PROCEEDINGS OF THE

AMERICAN MATHEMATICAL SOCIETY

Volume 128, Number 8, Pages 2213-2222

S 0002-9939(99)05570-7

Article electronically published on December 8, 1999

\title{
GENERIC POLYNOMIALS FOR QUASI-DIHEDRAL, DIHEDRAL AND MODULAR EXTENSIONS OF ORDER 16
}

\author{
ARNE LEDET
}

(Communicated by David E. Rohrlich)

\begin{abstract}
We describe Galois extensions where the Galois group is the quasidihedral, dihedral or modular group of order 16, and use this description to produce generic polynomials.
\end{abstract}

\section{INTRODUCTION}

Let $K$ be a field of characteristic $\neq 2$. Then every quadratic extension of $K$ has the form $K(\sqrt{a}) / K$ for some $a \in K^{*}$. Similarly, every cyclic extension of degree 4 has the form $K\left(\sqrt{r\left(1+c^{2}+\sqrt{1+c^{2}}\right)}\right) / K$ for suitable $r, c \in K^{*}$. In other words: A quadratic extension is the splitting field of a polynomial $X^{2}-a$, and a $C_{4}$-extension is the splitting field of a polynomial $X^{4}-2 r\left(1+c^{2}\right) X^{2}+r^{2} c^{2}\left(1+c^{2}\right)$, for suitably chosen $a, c$ and $r$ in $K$. This makes the polynomials $X^{2}-t$ and $X^{4}-2 t_{1}\left(1+t_{2}^{2}\right) X^{2}+t_{1}^{2} t_{2}^{2}\left(1+t_{2}^{2}\right)$ generic according to the following

Definition. Let $K$ be a field and $G$ a finite group, and let $t_{1}, \ldots, t_{n}$ and $X$ be indeterminates over $K$. A polynomial $F\left(t_{1}, \ldots, t_{n}, X\right) \in K\left(t_{1}, \ldots, t_{n}\right)[X]$ is called a generic (or versal) polynomial for $G$-extensions over $K$, if it has the following properties:

(1) The splitting field of $F\left(t_{1}, \ldots, t_{n}, X\right)$ over $K\left(t_{1}, \ldots, t_{n}\right)$ is a $G$-extension.

(2) If $L / K$ is a field extension, any $G$-extension of $L$ is obtained as the splitting field of $F\left(a_{1}, \ldots, a_{n}, X\right)$ for suitable $a_{1}, \ldots, a_{n} \in L$.

Generic polynomials (and the closely related generic Galois extensions; cf. $\underline{\mathrm{Sa}}$ ) are a convenient way of describing what $G$-extensions look like.

In this paper, we construct generic polynomials for the quasi-dihedral, dihedral and modular group of order 16 over fields of characteristic $\neq 2$. Here, the quasidihedral group of order 16 is the group $Q D_{8}$ with generators $u$ and $v$ and relations $u^{4}=v^{2}$ and $v u=u^{3} v$, the dihedral group of order 16 is the group $D_{8}$ with generators $\sigma$ and $\tau$ and relations $\sigma^{8}=\tau^{2}=1$ and $\tau \sigma=\sigma^{7} \tau$, and the modular group of order 16 is the group $M_{16}$ with generators $u$ and $v$ and relations $u^{8}=v^{2}=1$ and $v u=u^{5} v$.

Received by the editors September 8, 1998.

2000 Mathematics Subject Classification. Primary 12F12.

This work was supported by a Queen's University Advisory Research Committee Postdoctoral Fellowship. 
The approach is as follows: We start with a Galois extension $M / K$ of degree 8 , where the Galois group $G=\operatorname{Gal}(M / K)$ is a homomorphic image of the group $E$ $\left(=Q D_{8}, D_{8}\right.$ or $\left.M_{16}\right)$ we consider. This gives us a Galois theoretical embedding problem: Can we extend this $G$-extension to an E-extension? And if so, how? For the embedding problems we get, the criterion for solvability is that the crossed product algebra $(M, G, c)$ splits, where $c$ is a factor system representing the group extension

$$
1 \rightarrow \mu_{2} \rightarrow E \rightarrow G \rightarrow 1 .
$$

For a proof of this, see e.g. [Ki]. In all three cases, this algebra is a tensor product of two quaternion algebras and a matrix algebra, meaning that the criterion can be reformulated as an equivalence of quadratic forms. Details on how to find the obstruction can be found in [Le1], and the main reference for this paper is [Le2], where conditions in terms of quadratic forms are given, and solutions to the embedding problems are constructed.

It should be pointed out that the obstructions to realising $Q D_{8}$ given in Le1. Ex. 4.1] and in [Le2, 2.4] are not identical, since different maps $Q D_{8} \rightarrow D_{4}$ are used. (The more natural map is the one used in [Le1], as well as in [Ki]. On the other hand, for constructing the solutions the map used in [Le2] is more convenient.) However, the obstruction in [Le2] can be obtained directly from [Le1, Prop. 4.2]. For the other two embedding problems, the obstructions in [Le1] and Le2] are identical, although they have been rewritten slightly to accommodate the quadratic forms approach. This rewriting was done using $(a,-b)=1$ (for $\left.D_{8}\right)$ and $(a,-1)=1$ (for $M_{16}$ ).

Remark. In [Bl, Thm. 4.6], Black proves the existence of generic $D_{8}$-extensions, although a generic polynomial is not explicitly constructed. Indeed, the idea of this paper - using the descriptions of $Q D_{8^{-}}, D_{8^{-}}$and $M_{16^{-}}$extensions given in [Le2] to produce generic polynomials - was directly inspired by Black's result.

We let $D_{4}$ denote the dihedral group of order 8 , i.e., the group with generators $\sigma$ and $\tau$ and relations $\sigma^{4}=\tau^{2}=1$ and $\tau \sigma=\sigma^{3} \tau$. Also, we assume all fields to have characteristic $\neq 2$.

\section{THE QUASI-DIHEDRAL GROUP}

Let $M / K$ be a $D_{4}$-extension. By [Ki, Thm. 5], we may assume

$$
M=K(\sqrt{r(a+\sqrt{a})}, \sqrt{b}),
$$

where $a$ and $b=a-1$ in $K^{*}$ are quadratically independent, and $r \in K^{*}$ is arbitrary 1 Now, by [Le2, 2.4], $M / K$ can be embedded in a $Q D_{8}$-extension $F / K$, such that $F / K(\sqrt{b})$ is cyclic and $F / K(\sqrt{a} \sqrt{b})$ is dihedral, if and only if the quadratic forms $\langle b, 2 r a, 2 r a b\rangle$ and $\langle a, 2,2 a\rangle$ are equivalent over $K$. Thus, the embedding problem is solvable for some $r \in K^{*}$, if and only if the quadratic form $\langle a, 2,2 a\rangle$ represents $b$, i.e., if and only if

$$
a x^{2}+2 y^{2}+2 a z^{2}=b=a-1
$$

\footnotetext{
${ }^{1}$ In Ki], Kiming lists two kinds of $D_{4}$-extensions, the other being $K(\sqrt[4]{a}, \sqrt{-1}) / K$. However, the first kind, described above, covers everything.
} 
for suitable $x, y, z \in K$. Considering $y^{2}+a z^{2}$ as a norm in the quadratic extension $K(\sqrt{-a}) / K$ and multiplying $y+z \sqrt{-a}$ by a factor $(u+v \sqrt{-a}) /(u-v \sqrt{-a})$, we see that we can replace $y$ and $z$ by

$$
y^{\prime}=\frac{\left(u^{2}-a v^{2}\right) y-2 a u v z}{u^{2}+a v^{2}}, \quad z^{\prime}=\frac{\left(u^{2}-a v^{2}\right) z+2 u v y}{u^{2}+a v^{2}}
$$

for $u, v \in K$ with $u^{2}+a v^{2} \neq 0$, if necessary. (The fact that $-a$ may be a square in $K$ does not change the validity of this substitution.) Thus, we may assume $1-x^{2}-2 z^{2} \neq 0$ and get

$$
a=\frac{1+2 y^{2}}{1-x^{2}-2 z^{2}} .
$$

Choosing $u$ and $v$ properly, we may assume $a x^{2}+2 y^{2} \neq 0$ as well. Now,

$$
\mathbf{Q}^{t}\langle a, 2,2 a\rangle \mathbf{Q}=\left\langle b, 2 a\left(a x^{2}+2 y^{2}\right), 2 a b\left(a x^{2}+2 y^{2}\right)\right\rangle
$$

for

$$
\mathbf{Q}=\left(\begin{array}{ccc}
x & -2 y & -2 a x z \\
y & a x & -2 a y z \\
z & 0 & a x^{2}+2 y^{2}
\end{array}\right)
$$

Also, $\operatorname{det} \mathbf{Q}=b\left(a x^{2}+2 y^{2}\right)$.

Thus, the embedding problem is solvable for $r=a x^{2}+2 y^{2}$. More generally, it is solvable whenever $\langle b, 2 r a, 2 r a b\rangle \sim\left\langle b, 2 a\left(a x^{2}+2 y^{2}\right), 2 a b\left(a x^{2}+2 y^{2}\right)\right\rangle$. By the Witt Cancellation Theorem (see e.g. [Ja, 6.5 p. 367]) this is equivalent to $\langle 2 r a, 2 r a b\rangle \sim$ $\left\langle 2 a\left(a x^{2}+2 y^{2}\right), 2 a b\left(a x^{2}+2 y^{2}\right)\right\rangle$, i.e., to $\langle r, r b\rangle \sim\left(a x^{2}+2 y^{2}\right)\langle 1, b\rangle$. Hence, we must have $r=\left(a x^{2}+2 y^{2}\right)\left(p^{2}+b q^{2}\right)$ for suitable $p, q \in K$. And since we can modify $r$ by a factor from $K^{*} \cap\left(K(\sqrt{a}, \sqrt{b})^{*}\right)^{2}$ without changing $M$, we can assume $p=1$ and $r=\left(a x^{2}+2 y^{2}\right)\left(1+b q^{2}\right)$. Then

$$
\mathbf{Q}^{\prime t}\langle a, 2,2 a\rangle \mathbf{Q}^{\prime}=\langle b, 2 r a, 2 r a b\rangle
$$

when

$$
\mathbf{Q}^{\prime}=\mathbf{Q}\left(\begin{array}{ccc}
1 & 0 & 0 \\
0 & 1 & -b q \\
0 & q & 1
\end{array}\right)=\left(\begin{array}{ccc}
x & -2(y+a q x z) & 2(b q y-a x z) \\
y & a(x-2 q y z) & -a(b q x+2 y z) \\
z & \left(a x^{2}+2 y^{2}\right) q & a x^{2}+2 y^{2}
\end{array}\right)
$$

and $\operatorname{det} \mathbf{Q}^{\prime}=r b$.

The construction of $Q D_{8}$-extensions in [Le2] 2.4] uses the matrix $\mathbf{P}=\mathbf{Q}^{\prime-1}$ : If $\mathbf{P}^{t}\langle b, 2 r a, 2 r a b\rangle \mathbf{P}=\langle a, 2,2 a\rangle$ and $\operatorname{det} \mathbf{P}=1 / r b$, the $Q D_{8}$-extensions we seek are

$$
K(\sqrt{s \omega}, \sqrt{a}) / K, \quad s \in K^{*},
$$

where

$$
\begin{aligned}
\omega=1+p_{11} \sqrt{b} & / \sqrt{a}+\frac{1}{2}\left[p_{22}+p_{23} / \sqrt{a}-p_{32} \sqrt{b}+p_{33} \sqrt{b} / \sqrt{a}\right] \sqrt{r(a+\sqrt{a})} \\
+ & \frac{1}{2}\left[p_{22}-p_{23} / \sqrt{a}+p_{32} \sqrt{b}+p_{33} \sqrt{b} / \sqrt{a}\right] \frac{\sqrt{a}-1}{\sqrt{b}} \sqrt{r(a+\sqrt{a})} .
\end{aligned}
$$

Moreover, $K(\sqrt{s \omega}, \sqrt{a}) / K$ is the Galois closure of $K(\sqrt{s \omega}) / K$. 
Fortunately, it is easy to invert $\mathbf{Q}^{\prime}$ :

$$
\begin{aligned}
\mathbf{P} & =\mathbf{Q}^{\prime-1}=\langle 1 / b, 1 / 2 r a, 1 / 2 r a b\rangle \mathbf{Q}^{\prime t}\langle a, 2,2 a\rangle \\
& =\left(\begin{array}{ccc}
a x / b & 2 y / b & 2 a z / b \\
-(y+a q x z) / r & (x-2 q y z) / r & \left(a x^{2}+2 y^{2}\right) q / r \\
(b q y-a x z) / r b & -(b q x+2 y z) / r b & \left(a x^{2}+2 y^{2}\right) / r b
\end{array}\right) .
\end{aligned}
$$

We now have

Theorem 1. A $Q D_{8}$-extension has the form

$$
K(\sqrt{s \omega}, \sqrt{a}) / K, \quad s \in K^{*},
$$

where

$$
a=\frac{1+2 y^{2}}{1-x^{2}-2 z^{2}}
$$

for suitable $x, y, z \in K$, such that $a$ and $b=a-1$ are well-defined and quadratically independent, $a x^{2}+2 y^{2} \neq 0$, and

$$
\begin{aligned}
& \omega=+\frac{x \sqrt{a}}{\sqrt{b}} \\
&+\frac{1}{2 r}\left[x-2 q y z+\frac{q\left(a x^{2}+2 y^{2}\right)}{\sqrt{a}}+\frac{b q x+2 y z}{\sqrt{b}}+\frac{a x^{2}+2 y^{2}}{\sqrt{a} \sqrt{b}}\right] \sqrt{r(a+\sqrt{a})} \\
&+\frac{1}{2 r}\left[x-2 q y z-\frac{q\left(a x^{2}+2 y^{2}\right)}{\sqrt{a}}-\frac{b q x+2 y z}{\sqrt{b}}+\frac{a x^{2}+2 y^{2}}{\sqrt{a} \sqrt{b}}\right] \frac{\sqrt{a}-1}{\sqrt{b}} \sqrt{r(a+\sqrt{a})}
\end{aligned}
$$

for $q \in K$, such that $r=\left(a x^{2}+2 y^{2}\right)\left(1+b q^{2}\right) \neq 0$.

In particular, we get a $Q D_{8}$-extension over $K(x, y, z, q, s)$, when we consider $x$, $y, z, q$ and $s$ as indeterminates. This gives us our generic polynomial for $Q D_{8^{-}}$ extensions:

Theorem 2. Let $x, y, z, q$ and $s$ be indeterminates over the field $K$. Then the polynomial

$$
F(x, y, z, q, s, T)=\left(T^{2}-s\right)^{4}+s^{2} c_{2}\left(T^{2}-s\right)^{2}+s^{3} c_{1}\left(T^{2}-s\right)+s^{4} c_{0}
$$

in $K(x, y, z, q, s, T)$ is a generic polynomial for $Q D_{8}$-extensions over $K$, when

$$
\begin{gathered}
a=\frac{1+2 y^{2}}{1-x^{2}-2 z^{2}}, \quad b=a-1, \quad r=\left(a x^{2}+2 y^{2}\right)\left(1+b q^{2}\right), \\
\quad h=p_{23}+a p_{32}-p_{33}, \quad k=p_{22}-p_{32}+p_{33}, \\
\alpha=r\left(h^{2}+a k^{2}+2 h k\right) / 4, \quad \beta=r\left(h^{2}+a k^{2}+2 a h k\right) / 4 a \\
c_{2}=-2\left(a x^{2} / b+2 \alpha\right), \quad c_{1}=2 r x\left(p_{23}^{2}+a b p_{32}^{2}-a p_{22}^{2}-b p_{33}^{2}\right. \\
\left.\quad-2 a p_{22} p_{33}+2 a p_{23} p_{32}-2 p_{23} p_{33}+2 a p_{22} p_{32}\right), \\
c_{0}=a^{2} x^{4} / b^{2}+2\left(\alpha^{2}+a \beta^{2}\right)-4 a x^{2} \alpha / b-2\left(\alpha^{2}-a \beta^{2}\right)
\end{gathered}
$$

and the $p_{i j}$ 's are the entries in the matrix $\mathbf{P}$ above. Specifically, $Q D_{8}$-extensions are obtained by specialisations such that $a$ and $b$ are well-defined and quadratically independent, and $r$ and $s$ are $\neq 0$. 
Proof. $f(x, y, z, q, T)=T^{4}+c_{2} T^{2}+c_{1} T+c_{0}$ is the minimal polynomial for $\omega-$ 1 , where $\omega$ is as in Theorem 1. It follows that $F(x, y, z, q, s, T)$ is the minimal polynomial for $\sqrt{s \omega}$.

Remark. A few observations about the calculation of $f(x, y, z, q, T)$ are in order: Since $\theta=\omega-1$ has degree 4 and is a primitive element for the $C_{2} \times C_{2}$-extension $M / K(\sqrt{a})$, we are left with calculating minimal polynomials in $C_{2} \times C_{2}$-extensions:

Let $L / k=k(\sqrt{A}, \sqrt{B}) / k$ be a $C_{2} \times C_{2}$-extension, and let $\theta=a_{1} \sqrt{A}+a_{2} \sqrt{B}+$ $a_{3} \sqrt{A} \sqrt{B}, a_{1}, a_{2}, a_{3} \in k$, have degree 4 . Then the minimal polynomial for $\theta$ over $k$ is

$$
\begin{aligned}
f(T)=T^{4}- & 2\left(a_{1}^{2} A+a_{2}^{2} B+a_{3}^{2} A B\right) T^{2}-8 a_{1} a_{2} a_{3} A B T \\
& +\left(a_{1}^{4} A^{2}+a_{2}^{4} B^{2}+a_{3}^{4} A^{2} B^{2}-2 a_{1}^{2} a_{2}^{2} A B-2 a_{1}^{2} a_{3}^{2} A^{2} B-2 a_{2}^{2} a_{3}^{2} A B^{2}\right) .
\end{aligned}
$$

We notice that the coefficients in degrees 0 and 2 are expressed in terms of $a_{1}^{\prime}=a_{1}^{2} A$, $a_{2}^{\prime}=a_{2}^{2} B$ and $a_{3}^{\prime}=a_{3}^{2} A B$.

In the case of Theorem 2, we have $L / k=M / K(\sqrt{a}), A=b$ and $B=r(a+\sqrt{a})$. Also,

$$
\begin{aligned}
& a_{1}=p_{11} / \sqrt{a}, \\
& a_{2}=\frac{1}{2}\left[p_{22}+p_{23} / \sqrt{a}+p_{32}(\sqrt{a}-1)+p_{33}(\sqrt{a}-1) / \sqrt{a}\right], \quad \text { and } \\
& a_{3}=\frac{1}{2}\left[p_{22}(\sqrt{a}-1) / b-p_{23}(\sqrt{a}-1) / b \sqrt{a}-p_{32}+p_{33} / \sqrt{a}\right]
\end{aligned}
$$

Calculations (performed in Maple V) show that $a_{2}^{\prime}=r(1+\sqrt{a})(h+k \sqrt{a})^{2} / 4 \sqrt{a}=$ $\alpha+\beta \sqrt{a}$ and $a_{3}^{\prime}$ are conjugate in $K(\sqrt{a}) / K$. This simplifies the expressions for $c_{0}$ and $c_{2}$.

\section{THE DIHEDRAL GROUP}

Again, we look at a $D_{4}$-extension $M=K(\sqrt{r(a+\sqrt{a})}, \sqrt{b})$, where $b=a-1$. By [Le2, 3.3], $M / K$ can be embedded in a $D_{8}$-extension $F / K$, such that $F / K(\sqrt{b})$ is cyclic, if and only if the quadratic forms $\langle b, r a, r a b\rangle$ and $\langle a b, 2 a, 2 b\rangle$ are equivalent over $K$, and if $\mathbf{P}$ is a $3 \times 3$ matrix over $K$ with $\mathbf{P}^{t}\langle b, r a, r a b\rangle \mathbf{P}=\langle a b, 2 a, 2 b\rangle$ and $\operatorname{det} \mathbf{P}=2 / r$, the $D_{8}$-extensions in question are

$$
K(\sqrt{s \omega}, \sqrt{b}) / K, \quad s \in K^{*},
$$

where

$$
\begin{aligned}
& \omega=1-p_{11} / \sqrt{a} \\
& +\frac{1}{2}\left(p_{32}+p_{23} / \sqrt{a}\right) \sqrt{r(a+\sqrt{a})}+\frac{1}{2}\left(p_{22} / b-p_{33} / \sqrt{a}\right)(\sqrt{a}-1) \sqrt{r(a+\sqrt{a})} .
\end{aligned}
$$

Also, $K(\sqrt{s \omega}, \sqrt{b}) / K$ is the Galois closure of $K(\sqrt{s \omega}) / K$.

The embedding problem is solvable for some $r \in K^{*}$ if and only if $\langle a b, 2 a, 2 b\rangle$ represents $b$, i.e., if and only if the quadratic form $\langle a b, 2 a, 2 b,-b\rangle$ is isotropic. Multiplying by $2 a b$ and removing square factors, we see that this is equivalent to $\langle 2, b, a,-2 a\rangle$ being isotropic, or to $\langle a, 2,-2 a\rangle$ representing $-b$ :

$$
a x^{2}+2 y^{2}-2 a z^{2}=-b=1-a
$$


for suitable $x, y, z \in K$. We may assume $1+x^{2}-2 z^{2} \neq 0$ and get

$$
a=\frac{1-2 y^{2}}{1+x^{2}-2 z^{2}} .
$$

Modifying $y$ and $z$ properly, we may assume $z$ and $b+2 y^{2}$ to be non-zero as well.

Now, returning to the first criterion given,

$$
\mathbf{Q}^{t}\langle a b, 2 a, 2 b\rangle \mathbf{Q}=\left\langle b, a\left(b+2 y^{2}\right), a b\left(b+2 y^{2}\right)\right\rangle
$$

for

$$
\mathbf{Q}=\left(\begin{array}{ccc}
y / a z & -1 & -x y / z \\
b / 2 a z & y & -b x / 2 z \\
x / 2 z & 0 & \left(b+2 y^{2}\right) / 2 z
\end{array}\right)
$$

Also, $\operatorname{det} \mathbf{Q}=\left(b+2 y^{2}\right) / 2$.

Thus, the embedding problem is solvable for $r=b+2 y^{2}$, and more generally whenever $\langle r, r b\rangle \sim\left\langle b+2 y^{2}, b\left(b+2 y^{2}\right)\right\rangle$. Hence, we must have $r=\left(b+2 y^{2}\right)\left(p^{2}+b q^{2}\right)$ for suitable $p, q \in K$. Again, we can assume $p=1$ and thus $r=\left(b+2 y^{2}\right)\left(1+b q^{2}\right)$. Then

$$
\mathbf{Q}^{\prime t}\langle a b, 2 a, 2 b\rangle \mathbf{Q}^{\prime}=\langle b, r a, r a b\rangle
$$

when

$$
\mathbf{Q}^{\prime}=\mathbf{Q}\left(\begin{array}{ccc}
1 & 0 & 0 \\
0 & 1 & -b q \\
0 & q & 1
\end{array}\right)=\left(\begin{array}{ccc}
y / a z & -(z+q x y) / z & (b q z-x y) / z \\
b / 2 a z & (2 y z-b q x) / 2 z & -(2 q y z+x) b / 2 z \\
x / 2 z & \left(b+2 y^{2}\right) q / 2 z & \left(b+2 y^{2}\right) / 2 z
\end{array}\right)
$$

and $\operatorname{det} \mathbf{Q}^{\prime}=r / 2$.

We need to invert $\mathbf{Q}^{\prime}$, and again this is easy:

$$
\begin{aligned}
\mathbf{P} & =\mathbf{Q}^{\prime-1}=\langle 1 / b, 1 / r a, 1 / r a b\rangle \mathbf{Q}^{\prime t}\langle a b, 2 a, 2 b\rangle \\
& =\left(\begin{array}{ccc}
y / z & 1 / z & x / z \\
-b(z+q x y) / r z & (2 y z-b q x) / r z & \left(b+2 y^{2}\right) b q / r a z \\
(b q z-x y) / r z & -(x+2 q y z) / r z & \left(b+2 y^{2}\right) / r a z
\end{array}\right) .
\end{aligned}
$$

We now have

Theorem 3. A $D_{8}$-extension has the form

$$
K(\sqrt{s \omega}, \sqrt{b}) / K, \quad s \in K^{*}
$$

where

$$
a=\frac{1-2 y^{2}}{1+x^{2}-2 z^{2}}
$$

for suitable $x, y, z \in K$, such that $a$ and $b=a-1$ are well-defined and quadratically independent, $z$ and $b+2 y^{2}$ are non-zero, and

$$
\begin{array}{r}
\omega=1-\frac{y}{z \sqrt{a}}-\frac{2 a y z(1+b q)+a b(1-q) x+\left(b+2 y^{2}\right) b}{2 r a b z} \sqrt{r(a+\sqrt{a})} \\
+\frac{b\left(b+2 y^{2}\right)(1+b q)+a^{2}(2 y z-b q x)}{2 r a b z \sqrt{a}} \sqrt{r(a+\sqrt{a})}
\end{array}
$$

for $q \in K$, such that $r=\left(b+2 y^{2}\right)\left(1+b q^{2}\right) \neq 0$.

Considering $x, y, z, q$ and $s$ as indeterminates, we get our generic polynomial for $D_{8}$-extensions: 
Theorem 4. Let $x, y, z, q$ and $s$ be indeterminates over the field $K$. Then the polynomial

$$
G(x, y, z, q, s, T)=\left(T^{2}-s\right)^{4}+s^{2} d_{2}\left(T^{2}-s\right)^{2}+s^{3} d_{1}\left(T^{2}-s\right)+s^{4} d_{0}
$$

in $K(x, y, z, q, s, T)$ is a generic polynomial for $D_{8}$-extensions, when

$$
\begin{gathered}
a=\frac{1-2 y^{2}}{1+x^{2}-2 z^{2}}, \quad b=a-1, \quad r=\left(b+2 y^{2}\right)\left(1+b q^{2}\right), \\
\alpha=-y / a z, \quad \beta=-\left(2 a y z(1+b q)+a b(1-q) x+\left(b+2 y^{2}\right) b\right) / 2 r a b z \\
\gamma=\left(b\left(b+2 y^{2}\right)(1+b q)+a^{2}(2 y z-b q x)\right) / 2 r a^{2} b z \\
d_{2}=-2 a\left(\alpha^{2}+r \beta^{2}+r a \gamma^{2}+2 r \beta \gamma\right), \\
d_{1}=-4 r a \alpha\left(\beta^{2}+a \gamma^{2}+2 a \beta \gamma\right) \quad \text { and } \\
d_{0}=a\left(a \alpha^{4}+r^{2} b \beta^{4}+r^{2} a^{2} b \gamma^{4}-2 r a \alpha^{2} \beta^{2}-2 r a^{2} \alpha^{2} \gamma^{2}\right. \\
\left.-2 r^{2} a b \beta^{2} \gamma^{2}+2 r^{2} a \beta^{3} \gamma-4 r \alpha^{2} \beta \gamma\right) .
\end{gathered}
$$

Specifically, $D_{8}$-extensions are obtained by specialisations such that $a$ and $b$ are well-defined and quadratically independent, and $r$ and $s$ are $\neq 0$.

Proof. $g(x, y, z, q, T)=T^{4}+d_{2} T^{2}+d_{1} T+d_{0}$ is the minimal polynomial for $\omega-1$, where $\omega$ is as in Theorem 3 .

Remark. If $L / k=k(\sqrt{r(a+\sqrt{a})}) / k, a=1+c^{2}$, is a $C_{4}$-extension, the minimal polynomial for an element

$$
\theta=\alpha \sqrt{a}+\beta \sqrt{r(a+\sqrt{a})}+\gamma \sqrt{a} \sqrt{r(a+\sqrt{a})} \in M
$$

of degree 4 is

$$
\begin{aligned}
f(T)=T^{4}-2 a\left(\alpha^{2}+r \beta^{2}+r a \gamma^{2}+2 r \beta \gamma\right) T^{2} \\
-4 r a \alpha\left(\beta^{2}+a \gamma^{2}+2 a \beta \gamma\right) T+a\left(a \alpha^{2}+r^{2} c^{2} \beta^{4}+r^{2} c^{2} a^{2} \gamma^{4}\right. \\
\left.-2 r a \alpha^{2} \beta^{2}-2 r a^{2} \alpha^{2} \gamma^{2}-2 r^{2} c^{2} a \beta^{2} \gamma^{2}+2 r^{2} a \beta^{3} \gamma-4 r \alpha^{2} \beta \gamma\right) .
\end{aligned}
$$

In the case of Theorem 4 our $C_{4}$-extension is $L / k=M / K(\sqrt{b})$, and we let $\theta=\omega-1$ and $c=\sqrt{b}$. This gives us the minimal polynomial for $\omega-1$ over $K(\sqrt{b})$, and since $c=\sqrt{b}$ only occurs to the second power, the polynomial is in fact the minimal polynomial over $K$. Computing the minimal polynomial for $s \omega$ over $K$ is then trivial.

\section{THE MODULAR GROUP}

Let $M / K$ be a $C_{4} \times C_{2}$-extension. It is well-known that $C_{4}$-extensions have the form $K(\sqrt{r(a+\sqrt{a})}) / K$, where $a=1+c^{2}, c \in K^{*}$, is not a square, and $r \in K^{*}$ is arbitrary. Thus, we can write $M=K(\sqrt{r(a+\sqrt{a})}, \sqrt{b})$, where $a=1+c^{2}$ and $b \in K^{*}$ in $K^{*}$ are quadratically independent, and $r \in K^{*}$.

By [Le2, 3.5], $M / K$ can be embedded in an $M_{16}$-extension $F / K$, such that $F / K(\sqrt{a})$ is not cyclic, if and only if the quadratic forms $\langle 1,2 r a b, 2 r a b\rangle$ and $\langle a, 2 b, 2 a b\rangle$ are equivalent over $K$. So, in order for the embedding problem to 
be solvable for some $r \in K^{*}$, it is necessary and sufficient that the quadratic form $\langle a, 2 b, 2 a b\rangle$ represents 1, i.e.,

$$
a x^{2}+2 b y^{2}+2 a b z^{2}=1
$$

for suitable $x, y, z \in K$. We must have $y^{2}+a z^{2} \neq 0$, since otherwise $a x^{2}=1$, and so

$$
b=\frac{1-a x^{2}}{2\left(y^{2}+a z^{2}\right)} .
$$

Modifying $y$ and $z$ if necessary, we may assume $z$ and $a x^{2}+2 b(y / z)^{2}$ to be non-zero, and replacing $b$ by $b z^{2}, y$ by $y / z$ and $z$ by 1 , we get

$$
b=\frac{1-a x^{2}}{2\left(y^{2}+a\right)}
$$

and $a x^{2}+2 b y^{2} \neq 0$. Now,

$$
\mathbf{Q}^{t}\langle a, 2 b, 2 a b\rangle \mathbf{Q}=\left\langle 1,2 a b\left(a x^{2}+2 b y^{2}\right), 2 a b\left(a x^{2}+2 b y^{2}\right)\right\rangle
$$

for

$$
\mathbf{Q}=\left(\begin{array}{ccc}
x & -2 b y & -2 a b x \\
y & a x & -2 a b y \\
1 & 0 & a x^{2}+2 b y^{2}
\end{array}\right)
$$

and $\operatorname{det} \mathbf{Q}=a x^{2}+2 b y^{2}$.

Thus, the embedding problem is solvable for $r=a x^{2}+2 b y^{2}$, and more generally for $r=\left(a x^{2}+2 b y^{2}\right)\left(p^{2}+q^{2}\right)$ for $p, q \in K$ with $p^{2}+q^{2} \neq 0$. We can assume $p=1$ and $r=\left(a x^{2}+2 b y^{2}\right)\left(1+q^{2}\right)$. Then

$$
\mathbf{Q}^{\prime t}\langle a, 2 b, 2 a b\rangle \mathbf{Q}^{\prime}=\langle 1,2 r a b, 2 r a b\rangle
$$

when

$$
\mathbf{Q}^{\prime}=\mathbf{Q}\left(\begin{array}{ccc}
1 & 0 & 0 \\
0 & 1 & -q \\
0 & q & 1
\end{array}\right)=\left(\begin{array}{ccc}
x & -2 b(y+a q x) & 2 b(q y-a x) \\
y & a(x-2 b q y) & -a(q x+2 b y) \\
1 & \left(a x^{2}+2 b y^{2}\right) q & a x^{2}+2 b y^{2}
\end{array}\right),
$$

and $\operatorname{det} \mathbf{Q}^{\prime}=r$.

Using [Le2, 3.5], we get the $M_{16}$-extensions

$$
K(\sqrt{s \omega}, \sqrt{a}) / K, \quad s \in K^{*},
$$

where

$$
\begin{aligned}
\omega=1+p_{11} / \sqrt{a}+ & \frac{1}{2}\left[p_{22}+p_{23} / \sqrt{a}-p_{32}+p_{33} / \sqrt{a}\right] \sqrt{r(a+\sqrt{a})} \\
& +\frac{1}{2}\left[p_{22}-p_{23} / \sqrt{a}+p_{32}+p_{33} / \sqrt{a}\right] \frac{\sqrt{a}-1}{c} \sqrt{r(a+\sqrt{a})}
\end{aligned}
$$

where

$$
\begin{aligned}
\mathbf{P} & =\mathbf{Q}^{\prime-1}=\langle 1,1 / 2 r a b, 1 / 2 r a b\rangle \mathbf{Q}^{\prime t}\langle a, 2 b, 2 a b\rangle \\
& =\left(\begin{array}{ccc}
a x & 2 b y & 2 a b \\
-(y+a q x) / r & (x-2 b q y) / r & \left(a x^{2}+2 b y^{2}\right) q / r \\
(q y-a x) / r & -(q x+2 b y) / r & \left(a x^{2}+2 b y^{2}\right) / r
\end{array}\right) .
\end{aligned}
$$

Also, $K(\sqrt{s \omega}, \sqrt{b}) / K$ is the Galois closure of $K(\sqrt{s \omega}) / K$. 
Theorem 5. An $M_{16}$-extension has the form

$$
K(\sqrt{s \omega}, \sqrt{b}) / K, \quad s \in K^{*},
$$

where

$$
b=\frac{1-a x^{2}}{2\left(y^{2}+a\right)}
$$

for suitable $c, x, y \in K$, such that $a=1+c^{2}$ and $b$ are well-defined and quadratically independent, $a x^{2}+2 b y^{2} \neq 0$, and

$$
\begin{aligned}
\omega=1+ & x \sqrt{a} \\
& +\frac{1}{2 r}\left[x(1+q)+2 b y(1-q)+\frac{\left(a x^{2}+2 b y^{2}\right)(1+q)}{\sqrt{a}}\right] \sqrt{r(a+\sqrt{a})} \\
& +\frac{1}{2 r}\left[x(1-q)-2 b y(1+q)+\frac{\left(a x^{2}+2 b y^{2}\right)(1-q)}{\sqrt{a}}\right] \frac{\sqrt{a}-1}{c} \sqrt{r(a+\sqrt{a})}
\end{aligned}
$$

for $q \in K$, such that $r=\left(a x^{2}+2 b y^{2}\right)\left(1+q^{2}\right) \neq 0$.

Treating $c, x, y, q$ and $s$ as indeterminates, we then have

Theorem 6. Let $c, x, y, q$ and $s$ be indeterminates over the field $K$. Then the polynomial

$$
H(c, x, y, q, s, T)=\left(T^{2}-s\right)^{4}+e_{2}\left(T^{2}-s\right)^{2}+e_{1}\left(T^{2}-s\right)+e_{0}
$$

in $K(c, x, y, q, s, T)$ is a generic polynomial for $M_{16}$-extensions, when

$$
\begin{gathered}
a=1+c^{2}, \quad b=\frac{1-a x^{2}}{2\left(y^{2}+a\right)}, \quad r=\left(a x^{2}+2 b y^{2}\right)\left(1+q^{2}\right), \\
\beta=\left((c x+2 b y)(1+q)+\left(a x^{2}+2 b y^{2}+2 b c y-x\right)(1-q)\right) / 2 r c \\
\gamma=\left(\left(a c x^{2}+2 b c y^{2}-2 a b y\right)(1+q)+\left(a x(1-x)-2 b y^{2}\right)(1-q)\right) / 2 r a c \\
e_{2}=-2 a\left(x^{2}+r \beta^{2}+r a \gamma^{2}+2 r \beta \gamma\right), \\
e_{1}=-4 r a x\left(\beta^{2}+a \gamma^{2}+2 a \beta \gamma\right) \quad \text { and } \\
e_{0}=a\left(a x^{4}+r^{2} b \beta^{4}+r^{2} a^{2} b \gamma^{4}-2 r a x^{2} \beta^{2}-2 r a^{2} x^{2} \gamma^{2}\right. \\
\left.-2 r^{2} a b \beta^{2} \gamma^{2}+2 r^{2} a \beta^{3} \gamma-4 r x^{2} \beta \gamma\right) .
\end{gathered}
$$

Specifically, $M_{16}$-extensions are obtained by specialisations such that $a$ and $b$ are well-defined and quadratically independent, and $r$ and $s$ are $\neq 0$.

Proof. $h(c, x, y, q, T)=T^{4}+e_{2} T^{2}+e_{1} T+e_{0}$ is the minimal polynomial for $\omega-1$, where $\omega$ is as in Theorem 5 .

Remark. In [Le2], a description of $C_{8}$-extensions is produced from the description of $Q D_{8}$-extensions by, essentially, letting $b$ be a square. However, Saltman proves in [Sa, Thm. 5.11] that there is no generic $C_{8}$-extension over the rational numbers, and - by implication - no generic polynomial for $C_{8}$-extensions in that case either. The reason the construction of generic polynomials works for $Q D_{8}, D_{8}$ and $M_{16}$, but not for $C_{8}$, is the extra degree of freedom obtained by introducing $b$ : If we try to carry through the calculations for $C_{8}$, we get a condition of the type $a-1=$ $\left(1+2 y^{2}\right) /\left(1-x^{2}-2 z^{2}\right)-1=c^{2}$, and it is not clear how to ensure that $a-1$ is a 
square, while at the same time getting enough $a$ 's. (Indeed, by Saltman's result it is impossible.) Thus, paradoxically, the larger groups $Q D_{8}, D_{8}$ and $M_{16}$ are easier to handle than the smaller group $C_{8}$.

\section{REFERENCES}

[Bl] E. V. Black, Deformations of Dihedral 2-Group Extensions of Fields, Trans. Amer. Math. Soc. 351 (1999), 3229-3241. MR 99m:12009

[Ja] N. Jacobson, Basic Algebra I, W. H. Freeman and Company, New York, 1985. MR 86d:00001

[Ki] I. Kiming, Explicit Classifications of some 2-Extensions of a Field of Characteristic different from 2, Canad. J. Math. 42 (1990), 825-855. MR 92c:11115

[Le1] A. Ledet, On 2-Groups as Galois Groups, Canad. J. Math. 47 (1995), 1253-1273. MR 97a:12003

[Le2] — Embedding Problems and Equivalence of Quadratic Forms, Math. Scand. (to appear).

[Sa] D. Saltman, Generic Galois Extensions and Problems in Field Theory, Adv. Math. 43 (1982), 250-283. MR 84a:13007

Department of Mathematics and Statistics, Queen's University, Kingston, Ontario, CANADA K7L 3N6

E-mail address: ledet@mast.queensu.ca 